\title{
Pneumonia necrotizante por Staphylococcus aureus resistente à meticilina
}

\author{
Necrotizing pneumonia due to methicillin-resistant \\ Staphylococcus aureus
}

\section{Pedro Alves d'Azevedo ${ }^{1,2}$, Fernanda Matsiko Inoue', Soraya Sgambatti Andrade', Regina Tranchesi ${ }^{3}$ e Antonio Carlos Campos Pignatari ${ }^{1}$}

\begin{abstract}
RESUMO
O objetivo desse estudo foi descrever um caso de pneumonia necrotizante por Staphylococcus aureus resistente a meticilina. A amostra foi isolada em hemocultura coletada menos de 48 horas da admissão hospitalar. A paciente era previamente hígida quando do início do processo infeccioso. 0 isolado possuía o gene mecA, com "staphylococcal cassette chromosome mec" tipo IVa". A presença de Staphylococcus aureus carreando esse determinante genético em nosso meio deve ser considerada em pneumonias comunitárias graves.
\end{abstract}

Palavras-chaves: Staphylococcus aureus. Resistência meticilina. Pneumonia necrotizante.

\section{ABSTRACT}

The aim of this study was to describe a case of necrotizing pneumonia caused by methicillin-resistant Staphylococcus aureus. The sample was isolated from a blood culture collected less than 48 hours after hospital admission. The patient had been healthy until the infectious process started. The isolate had the mecA gene with "staphylococcal cassette chromosome mec" (SCCmec) type IVa. The possibility that Staphylococcus aureus harboring this genetic determinant might be present in our setting should be considered in situations of severe pneumonia within the community.

Key-words: Staphylococcus aureus. Methicillin resistance. Necrotizing pneumonia.

Staphylococcus aureus resistentes a meticilina (MRSA) foram primeiramente descritos como microrganismos adquiridos em infecções nosocomiais ${ }^{1}$. No entanto, nos últimos anos, infecções de pele $^{156}$ e pneumonias necrotizantes ${ }^{34}$ causadas por MRSA adquiridos na comunidade (CA-MRSA) têm sido descritos em várias áreas geográficas ${ }^{7}$, principalmente nos Estados Unidos. A gravidade do quadro clínico em alguns casos está associada à produção da toxina de Panton-Valentine (PVL $)^{610}$, pela aquisição do gene $l u k S$-lukF. Resumindo, importantes características genéticas estão associadas com as cepas de CA-MRSA: a presença do elemento genético móvel denominado cassete cromossômico estafilocócico mec (SCCmec) tipo IV e V que transporta o gene $m e c A$ que codifica a proteína PBP2, que tem baixa afinidade por agentes antimicrobianos com anel betalactâmico, deixando assim de

\footnotetext{
1. Laboratório Especial de Microbiologia Clínica, Universidade Federal de São Paulo, São Paulo, SP. 2. Departamento de Ciências Básicas da Saúde da Universidade Federal de Ciências da Saúde de Porto Alegre, Porto Alegre, RS. 3. Comissão de Controle de Infecção do Hospital Nove de Julho, São Paulo, SP.

Apoio financeiro: $\mathrm{CNPq}$

Endereço para correspondência: Prof Pedro A. d'Azevedo. Dept ${ }^{0}$ de Ciências Básicas da Saúde/ UFCSPA. Rua Sarmento Leite 245/204, Centro, 90050-170 Porto Alegre, RS.

Tel: $55513303-8740$

e-mail: pedro_dazevedo@yahoo.com.br

Recebido para publicação em 26/11/2008

Aceito em 29/01/2009
}

ser efetivo e os genes da citotoxina Panton-Valentine leucocidine (PVL). No Brasil, os primeiros relatos de CA-MRSA, com estas características, causando infecções de pele, foram descritos por Ribeiro e cols ${ }^{8}$. 0 objetivo deste estudo foi descrever um caso de pneumonia necrotizante por CA-MRSA, no qual não se identificou a toxina PVL.

\section{RELATO DO CASO}

Paciente de 17 anos, previamente hígida, foi admitida em hospital da Cidade de São Paulo, em junho de 2006, com diagnóstico inicial de infecção de vias aéreas superiores. Em apenas 3 dias, evoluiu com piora do quadro clínico, broncopneumonia, choque séptico e insuficiência respiratória necessitando de ventilação mecânica.

Houve crescimento de cocos Gram-positivos em duas amostras de hemocultura coletada, em menos de 48 horas da admissão hospitalar. A amostra clínica foi identificada como MRSA pelo sistema automatizado AutoScan (DadeBehring) e confirmada por métodos tradicionais, sendo suscetível à sulfametoxazoltrimetoprima e clindamicina, e resistente à cefoxitina e eritromicina no teste de indução para detecção do fenótipo MSL (macrolídeos, lineosaminas e estreptograminas)negativo. A concentração inibitória mínima pela técnica de Etest (AB Biodisk, 
Solna, Suécia) foi de $3 \mu \mathrm{g} / \mathrm{ml}$ para oxacilina, sendo ainda suscetível a vancomicina $(2 \mu \mathrm{g} / \mathrm{mL})$ e teicoplanina $(2 \mu \mathrm{g} / \mathrm{mL})$. Pela técnica da reação em cadeia da polimerase, utilizando o protocolo de Zhang e cols ${ }^{11}$, a amostra foi caracterizada como mecA positiva, SCCmec (staphylococcal cassette chromosome me) tipo IVa com positividade para os genes de toxinas $\gamma$ hemolisina, enteroxina 0 (sea) e negativo para leucocidina Panton-Valentine.

A paciente evoluiu com cavitações pulmonares, empiema e fístula bronco-pleural, necessitando de drenagem pleural bilateral sendo submetida a traqueostomia no $11^{\circ}$ dia de internação. No primeiro dia de internação na unidade de terapia intensiva foi iniciado tratamento com hemofiltração e medidas de suporte hemodinâmico para o quadro de choque com melhora progressiva da insuficiência renal. No $16^{\circ}$ dia de internação, a paciente foi submetida à colectomia total com ileostomia por colite isquêmica. A antibioticoterapia foi mantida com teicoplanina (36 dias) recebendo também meropenem a partir do $7^{\circ}$ dia por 14 dias. A sorologia para HIV foi negativa. A paciente evoluiu com melhora progressiva, e alta após 72 dias de internação, ainda com drenagem pleural e ileostomia. Foram realizadas coletas de swab nasal em 3 membros familiares, porém em nenhum dos mesmos foi isolado MRSA.

\section{DISCUSSÃO}

Os genes de PVL codificam a produção de citotoxinas que causam necrose tecidual e destruição de leucócitos, através da formação de poros na membrana celular. A maioria das cepas de CA-MRSA portadoras desse gene está associada a infecções de pele, principalmente furúnculos e abcessos, mas pode também causar infecção tecidual necrotizante, pneumonia fulminante e sepse grave. 0 cassete cromossomal SCCmec IV encontrado nos isolados do CA-MRSA é pequeno em tamanho e perde genes de resistência apresentando assim suscetibilidade a várias classes de antimicrobianos não-betalactâmicos. No entanto, a regra para toxina PVL na patogênese do Staphylococcus aureus resistente a meticilina não está completamente elucidada, mas parece ser o fator de risco para infecções graves de pele e partes moles. A origem de isolados de CA-MRSA aconteceram no final da década de 80' na Austrália, entre os arborígenes daquele País e as amostras não apresentavam a toxina de Panton-Valentine.

Os isolados relatados nesse caso confirmaram serem SCCmec do tipo IVa, mas sem apresentar positivo a toxina PVL, apesar de serem encontradas outra importantes toxinas, a toxinas $\gamma$ hemolisina e a enteroxina 0 (sea), que podem ter contribuído para o quadro grave apresentado pela paciente.

A sensibilidade in vitro a sulfametoxazol-trimetropina e clindamicina é característica relevante descrita em CA-MRSA, particularmente em amostra $S C C m e c$ tipoIV ${ }^{3}{ }^{8}$. A resistência à oxacilina foi detectada inicialmente por disco difusão e confirmada pelo E-test com concentração inibitória mínima de $3 \mu \mathrm{g} / \mathrm{ml}$ próximo do breakpoint de $2 \mu \mathrm{g} / \mathrm{ml}^{2}$. A utilização terapêutica do glicopeptídeo teicoplanina precedeu a identificação bacteriana e o resultado do testes de sensibilidade. Foi utilizado um amplo esquema antimicrobiano inicial devido à gravidade do quadro e extensão do comprometimento pulmonar com descalonamento posterior, mantendo-se a terapêutica com teicoplanina com doses corrigidas para insuficiência renal. 0 meropenem foi utilizado a partir do sétimo dia de internação após a instalação de drenagem torácica bilateral considerando o risco de infecção por bacilos gram-negativos. A utilização de clindamicina e sulfametoxazol, além da linezolida poderia ser considerada além dos glicopeptídeos vancomicina e teicoplanina.

Concluindo, a presença de Staphylococcus aureus resistente a oxacilina, com esse determinante genético e sem carrear a toxina PVL, deve ser considerada em nosso meio em pneumonias comunitárias graves exigindo terapêutica antimicrobiana empírica adequada, vigilância epidemiológica rigorosa e diagnóstico laboratorial preciso, incluindo técnicas moleculares.

\section{REFERÊNCIAS}

1. Center for Disease Control and Prevention - Four pediatrics deaths from community-acquired methicillin-resistant Staphylococcus aureus - Minnesota and North Dakota, 1997-1999. Morbidity and Mortality Weekly Report 48:707$710,1999$.

2. Clinical and Laboratory Standards Institute. Performance Standards for Antimicrobial Susceptibility Testing; Seventeenth Informational Supplement. Approved Standard M100-S17. CLSI, Wayne, PA, USA, 2007.

3. Hageman JC, Uyeki TM, Francis JS, Jernigan DB, Wheeler JG, Bridges CB, Barenkamp SJ, Sievert DM, Srinivasan A, Doherty MC, McDougal LK, Killgore GE, Lopatin UA, Coffman R, McDonald JK, McAllister SK, Fosheim GE, Patel JB, McDonald LC. Severe community-acquired pneumonia due to Staphylococcus aureus, 200304 influenza season. Emerging Infectious Diseases 12:894-899, 2006.

4. Kallen AJ, Brunkard J, Moore Z, Budge P, Arnold KE, Fosheim G, Finelli L, Beekmann SE, Polgreen PM, Gorwitz R, Hageman J. Staphylococcus aureus community-adquired pneumonia during the 2006 to 2007 influenza season. Annals of Emergency Medicine 53:358-365, 2009.

5. Kazakova SV, Hageman JC, Matava M, Srinivasan A, Phelan L, Garfinkel B, Boo T, McAllister S, Anderson J, Jensen B, Dodson D, Lonsway D, McDougal LK, Arduino M, Fraser VJ, Killgore G, Tenover FC, Cody S, Jernigan DB. A clone of methicillin-resistant Staphylococcus aureus among professional football players. New England Journal of Medicine 352:28-35, 2005.

6. Larsen AR, Böcher S, Stegger M, Goering R, Pallesen LV, Skov R. Epidemiology of European Community-Associated Methicilin-Resistant Staphylococcus aureus clonal complex 80 type IV strains isolated in Denmark from 1993 to 2004. Journal of Clinical Microbiology 46:62-68, 2008.

7. Okuma K, Iwakawa K, Turnidge JD, Grubb WB, Bell JM, O'Brien FG, Coombs GW, Pearman JW, Tenover FC, Kapi M, Tiensasitorn C, Ito T, Hiramatsu K. Dissemination of new methicillin-resistant Staphylococcus aureus clones in the community. Journal of Clinical Microbiology 40:4289-4294, 2002.

8. Ribeiro A, Dias C, Silva-Carvalho MC, Berquó L, Ferreira FA, Santos RNS, FerreiraCarvalho B, Figueiredo AM. First report of infection with community-acquired methicillin-resistant Staphylococcus aureus in South America. Journal of Clinical Microbiology 43:1985-1988, 2005.

9. Udo EE, Pearmann JW, Grubb WB. Genetic análisis of community isolates of methicillin-resistant $S$. aureus in Western Australia. Journal of Hospital Infection 25:97-108, 1993

10. Vandenesch F, Naimi T, Enright MC, Lina G, Nimmo R, Hefferman H, Liassine N, Bes M, Greenland T, Reverdy ME, Etienne J. Community-acquired methicillinresistant Staphylococcus aureus carrying Panton-Valentine leukocidin genes: worldwide emergence. Emerging Infectious Diseases 9:978-984, 2003.

11. Zhang K, McClure JA, Elsayed S, Louie T, Conly JM. Novel Multiplex PCR assay for characterization and concomitant subttping of Staphylococcal cassette chromosome mec types I to V in methicillin-resistant Staphylococcus aureus. Journal of Clinical Microbiology 43:5026-5033, 2005. 